\title{
Neuroendocrine carcinoma of the lung expressing anaplastic lymphoma kinase on high-sensitivity immunohistochemistry: A case report
}

\author{
KOTOKO MIYOSHI ${ }^{1}$, YASUSHI ADACHI ${ }^{2-4}$, HITOSHI NAKAJI ${ }^{1}$, AKIHARU OKAMURA $^{5}$, \\ YASUHIRO SAKAI ${ }^{6}$, RYUJI HIRANO ${ }^{7}$, SHINSUKE YAHATA ${ }^{8}$, MING LI $^{3,9}$ and SUSUMU IKEHARA ${ }^{3,10}$
}

Departments of ${ }^{1}$ Respiratory Medicine and ${ }^{2}$ Diagnostic Pathology, Toyooka Hospital, Toyooka, Hyogo; Departments of ${ }^{3}$ Stem Cell Disorders and ${ }^{4}$ Pediatrics, Kansai Medical University, Hirakata, Osaka; ${ }^{5}$ Department of Diagnostic Pathology,

Kakogawa Central Hospital, Kakogawa; ${ }^{6}$ Department of Diagnostic Pathology, Kobe University Graduate School of Medicine/School of Medicine, Kobe; ${ }^{7}$ Division of Thoracic and Cardiovascular Surgery, Toyooka Hospital, Toyooka; ${ }^{8}$ Department of Internal General Medicine, Hamasaka Public Hospital, Hamasaka, Hyogo; ${ }^{9}$ Department of Laboratory for Cardiovascular Disease, Novel, Non-invasive and Nutritional Therapeutics (CNT), Osaka University, Suita, Osaka; ${ }^{10}$ Kansai Medical University, Hirakata, Osaka, Japan

Received November 9, 2016; Accepted March 29, 2017

DOI: $10.3892 /$ mco.2017.1308

\begin{abstract}
It has been reported that anaplastic lymphoma kinase $(A L K)$ protein is expressed in a proportion of non-small-cell carcinomas (mainly adenocarcinomas). By contrast, high-sensitivity immunohistochemistry (IHC) rarely detects ALK protein expression in neuroendocrine carcinomas (NECs) of the lung, which include small-cell carcinomas and large-cell neuroendocrine carcinomas (LCNECs). We herein present a case of NEC that was identified as ALK-positive via high-sensitivity IHC. A 51-year-old man was diagnosed with small-cell carcinoma in the upper lobe of the right lung. Although high-sensitivity IHC revealed that the tumor weakly expressed the ALK protein, no fusion gene with $A L K$ was found using fluorescence in situ hybridization (FISH). Standard chemotherapy was administered to the patient. Six months after the first visit to the hospital for the tumor, another tumor was identified in the upper lobe of the left lung. The tumor was resected and diagnosed as NEC displaying LCNEC-like characteristics. This NEC also moderately expressed ALK protein by high-sensitivity IHC, without exhibiting fusion genes with $A L K$ on FISH. These data suggest that the presence of $A L K$ fusion genes should be confirmed by FISH or reverse transcription polymerase chain reaction, even if high-sensitivity IHC for ALK protein is positive in lung cancer.
\end{abstract}

Correspondence to: Dr Yasushi Adachi, Department of Diagnostic Pathology, Toyooka Hospital, 1094 Tobera, Toyooka, Hyogo 668-8501, Japan

E-mail: adachiya250@gmail.com

Key words: lung, neuroendocrine carcinoma, anaplastic lymphoma kinase, high-sensitivity immunohistochemistry

\section{Introduction}

The expression of anaplastic lymphoma kinase (ALK) protein, induced by genomic fusion of the $A L K$ and nucleophosmin $(N P M)$ genes, was first reported in lymphoma cells (1). Such cases are referred to as ALK-positive anaplastic lymphomas. Subsequently, genomic alterations of $A L K$ were also reported for other tumors, including lung cancers, neuroblastomas and inflammatory myofibroblastic tumors (2-5). It has been reported that the percentage of genomic alterations of $A L K$ is $3-5 \%$ in lung cancers, and that the genomic alterations are mainly found in adenocarcinomas $(2,6)$. To date, several ALK tyrosine kinase inhibitors have been developed, such as crizotinib, certinib and alectinib, and ALK inhibitors have been found to be effective treatments for lung cancers with genomic alterations of $A L K$ (7). Several methods are currently used to detect $A L K$ genomic alterations in lung cancers in clinical practice, including reverse transcription polymerase chain reaction (RT-PCR), fluorescence in situ hybridization (FISH) and high-sensitivity immunohistochemistry (IHC). It has been reported that data from high-sensitivity IHC for ALK exhibit a good correlation with data from RT-PCR and FISH analyses $(8,9)$. Recently, high-sensitivity IHC tends to be performed first due to financial considerations. However, it has been reported that a small proportion of NECs of the lung express ALK protein on high-sensitivity IHC, without genomic alterations (10).

We herein present a case of NEC of the lung that was ALK-positive on high-sensitivity IHC, without the presence of fusion genes of $A L K$ on FISH.

\section{Case report}

A 51-year-old man visited Toyooka Hospital (Toyooka, Japan) in November 2010 due to a tumor in the right lung that was 


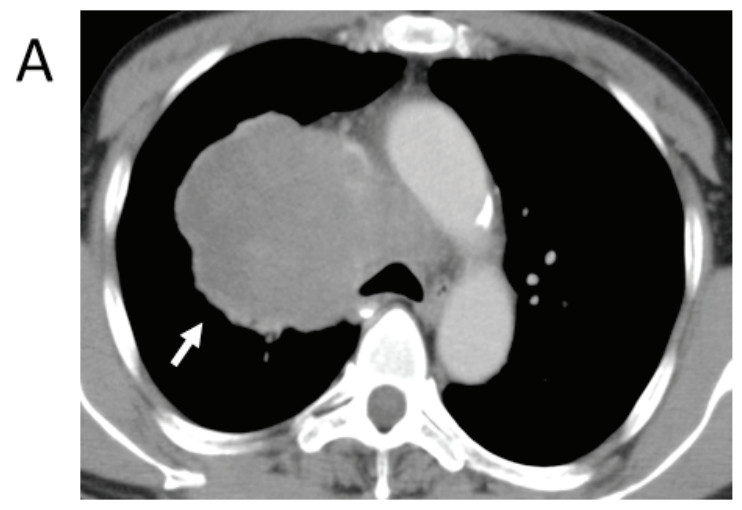

B
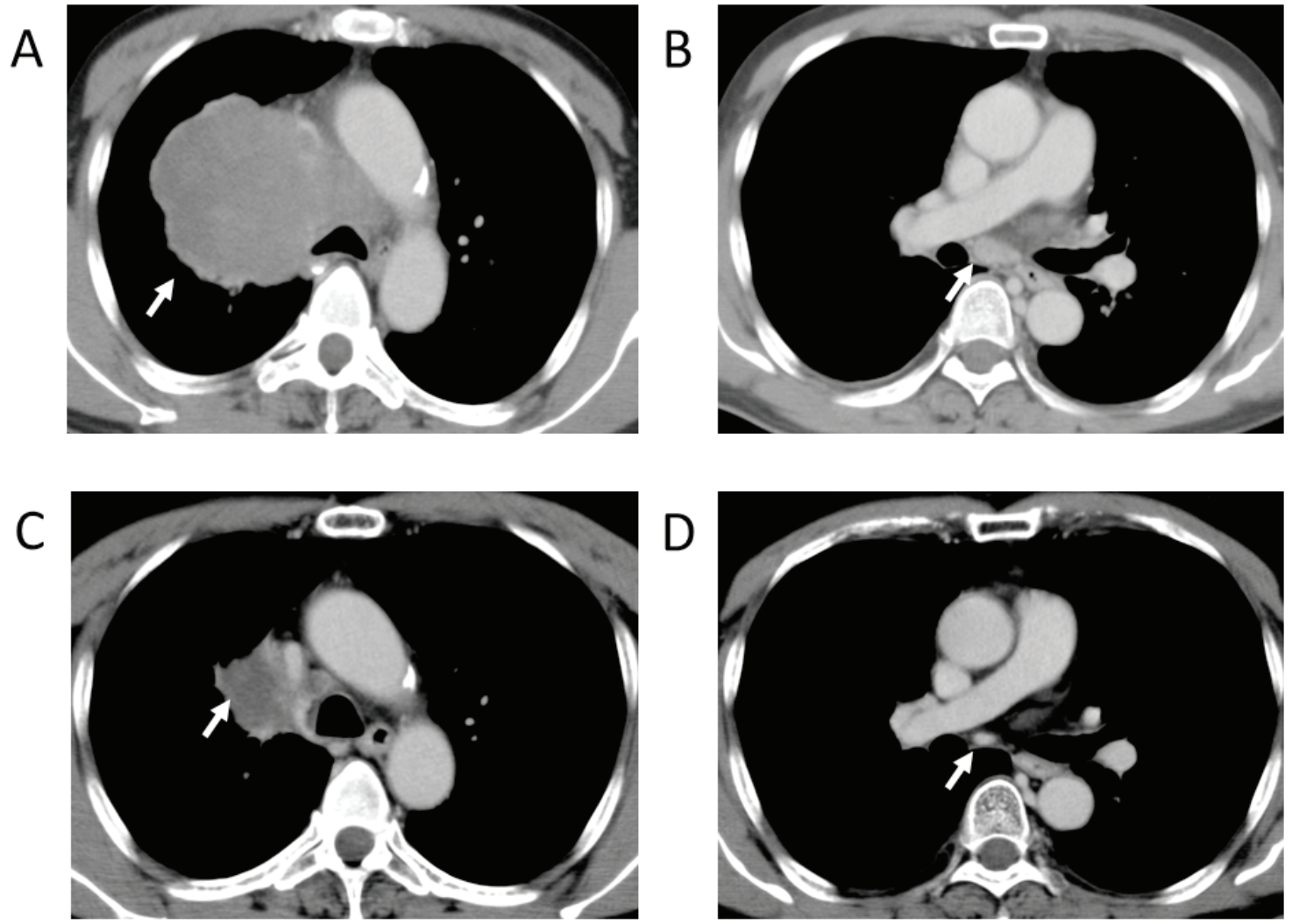

Figure 1. Computed tomography scan prior to and following chemotherapy. (A) A large tumor (diameter, $90 \mathrm{~mm}$ ) with low blood flow was identified in the hilum of the right lung (arrow). The tumor infiltrated into the superior vena cava. (B) An enlarged lymph node (diameter, $12 \mathrm{~mm}$ ) was detected at the bronchial bifurcation (arrow). After 3 courses of therapy, the sizes of (C) the tumor in the right lung and (D) the lymph node at the bronchial bifurcation were reduced (arrows).

A
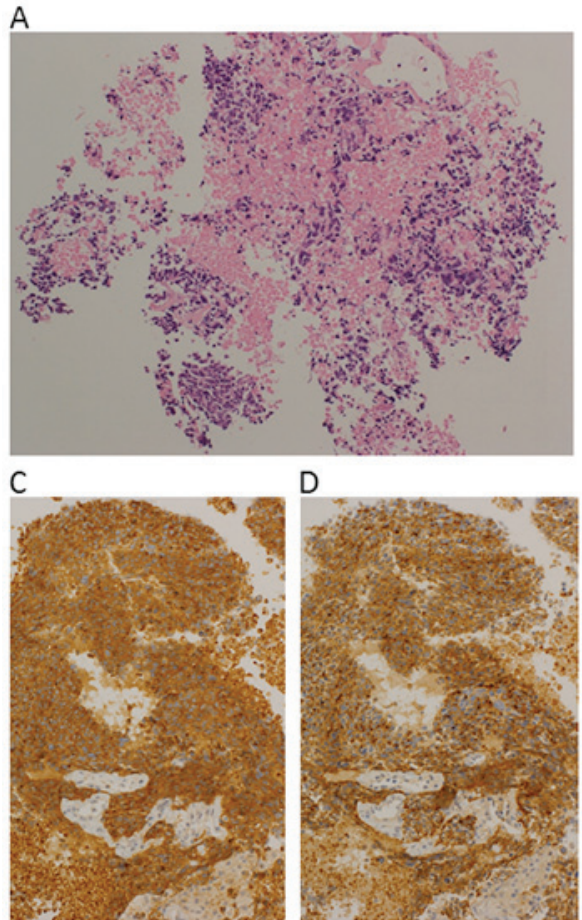

B
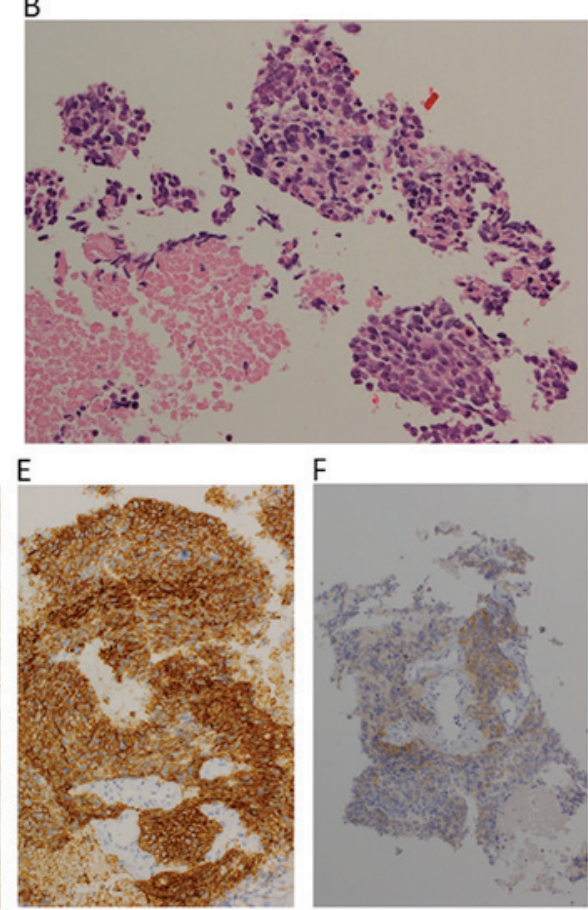

Figure 2. Histological analysis of the biopsied sample. The hematoxylin and eosin-stained specimen is shown at an original magnification of (A) x10 and (B) $x$ 40. The tumor cells expressed (C) synaptophysin, (D) chromogranin A, (E) CD56 and (F) anaplastic lymphoma kinase (magnification, x20).

identified on a chest X-ray during a routine annual checkup. The patient had hypertension and a history of several episodes of pancreatitis and he had previously undergone partial gastrectomy for gastric ulcer. The patient was a smoker 

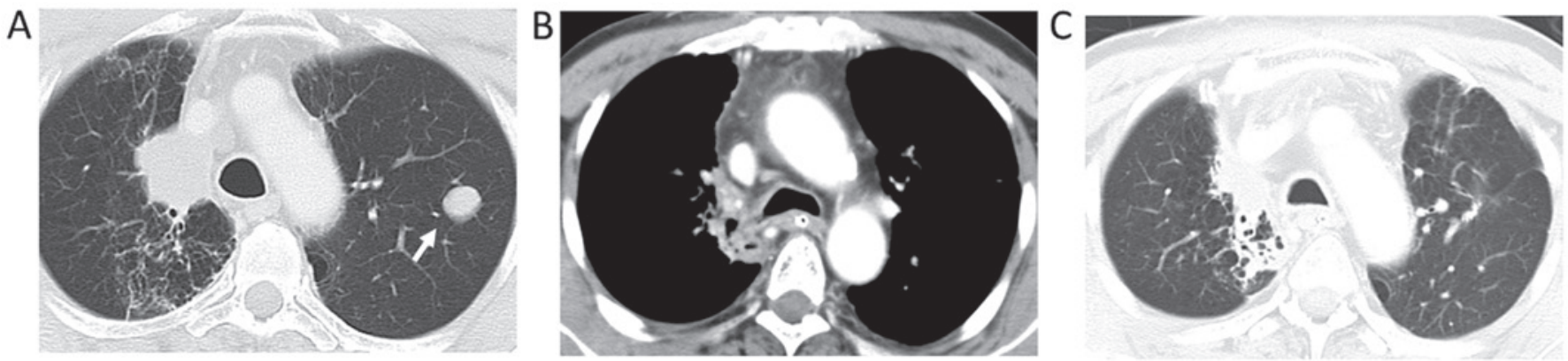

Figure 3. Computed tomography (CT) scan preformed 14 months after the patient's first visit to the hospital for the first neuroendocrine carcinoma (NEC) and for follow-up. (A) A second tumor (arrow) was identified in the upper lobe of the left lung 6 months after the patient's first visit to the hospital and gradually increased in size. The image shows the CT scan of prior to resection (14 months after the first visit to the hospital). (B) CT scan performed during follow-up for the first NEC in the hilum of the right lung, 4 years and 6 months after the patient's first visit to the hospital. The size of the first NEC arising in the right lung was significantly reduced. (C) Follow-up CT scan of the second NEC 4 years and 6 months after the patient's first visit to the hospital.

A

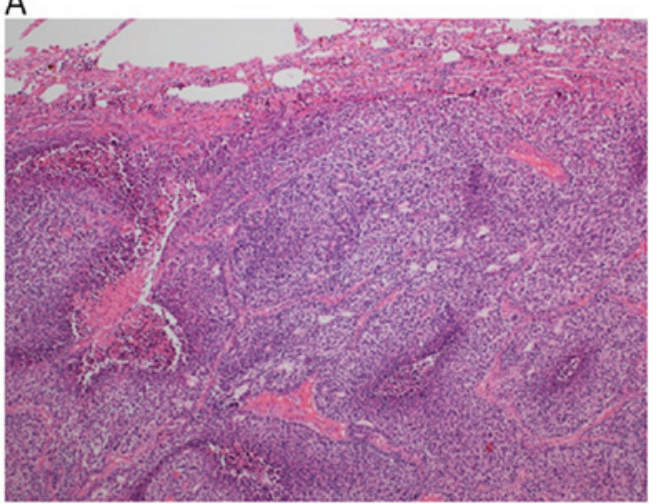

C

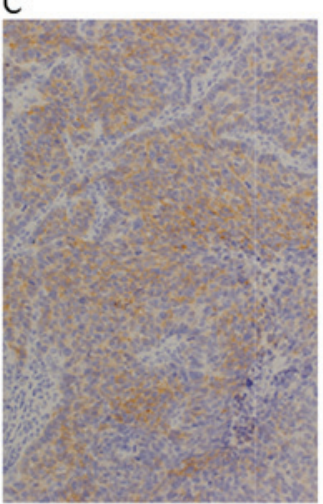

D

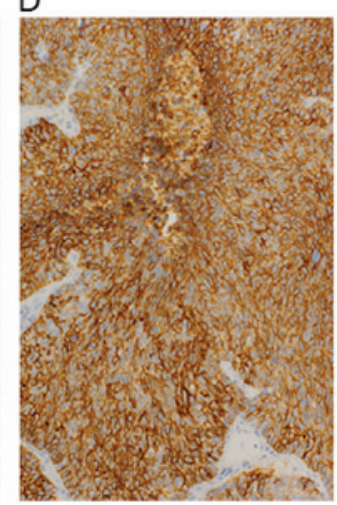

B

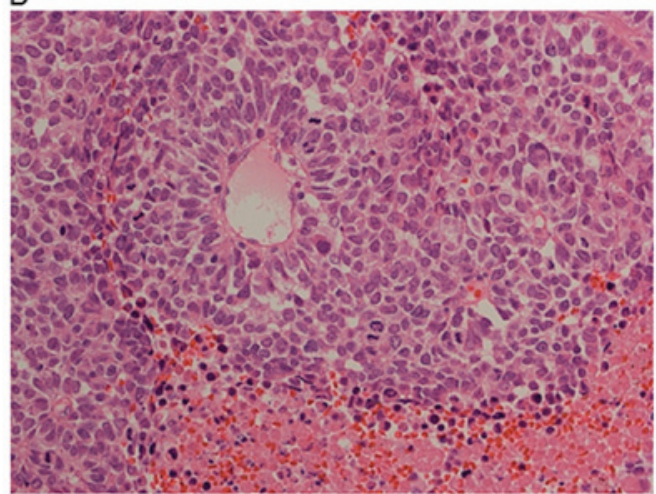

$\mathrm{E}$

$\mathrm{F}$

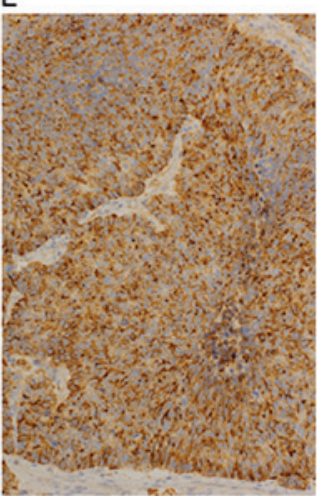

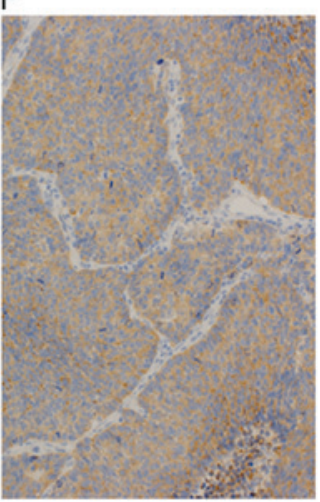

Figure 4. Histological analysis of the second tumor in the upper lobe of the left lung. The hematoxylin and eosin-stained specimen is shown at an original magnification of (A) x10 and (B) x40. The tumor cells expressed (C) synaptophysin, (D) chromogranin A, (E) CD56 and (F) anaplastic lymphoma kinase (magnification, $\mathrm{x} 20)$.

(20-30 cigarettes per day for 30 years) and consumed $30-90 \mathrm{ml}$ of alcohol per day.

Upon physical examination, superior vena cava syndrome was found. A chest computed tomography (CT) scan that was conducted during the first visit to the hospital revealed a tumor (90 $\mathrm{mm}$ in diameter) in the upper lobe of the right lung and enlargement of the lymph nodes in the hilar, peribronchial and peritracheal regions (Fig. 1A and B). A CT-guided percutaneous needle biopsy was performed. Histologically, the specimen contained tumor cells and necrotic tissue. The tumor cells were medium-sized and had a round-oval shape with minimal cytoplasm and hyperchromatic nuclei (Fig. 2A and B).
The tumor cells expressed synaptophysin, chromogranin A and CD56, suggesting the diagnosis of small-cell carcinoma (Fig. 2C, D and E). The tumor also weakly expressed ALK protein (Fig. 2F). However, FISH analysis did not reveal the presence of an $A L K$ fusion gene (data not shown).

The patient received chemotherapy with 6 courses of cisplatin $\left(80 \mathrm{mg} / \mathrm{m}^{2}\right.$ per day on day 1) plus etoposide $\left(100 \mathrm{mg} / \mathrm{m}^{2}\right.$ on days 1,2 and 3) and local radiation therapy (60 Gy/30 fractions). After 3 courses of chemotherapy, the sizes of the tumor and lymph nodes were reduced, suggesting partial remission (Fig. 1C and D). Six months after the patient's first visit to the hospital, another tumor was detected in the upper lobe of the 
left lung. As the tumor in the left lung gradually increased in size, it was resected via partial excision of the upper lobe of the left lung by thoracoscoscopic surgery that was performed 14 months after the patient's first visit to the hospital (Fig. 3A). The size of the resected tumor was $32 \times 25 \times 25 \mathrm{~mm}$ following formalin fixation. On histological examination, the tumor exhibited organoid nesting, trabecular growth, rosette-like structures and peripheral palisading. Rosette-like structures with a cribriform pattern were observed in the solid nests. Compared with small-cell carcinoma cells, these tumor cells contained abundant cytoplasm (Fig. 4A and B). The tumor cells were moderately positive for synaptophysin and clearly positive for chromogranin A and CD56 (Fig. 4C, D and E), suggesting the diagnosis of NEC with large-cell neuroendocrine carcinoma (LCNEC)-like characteristics. The tumor cells were also moderately positive for ALK on high-sensitivity IHC (Fig. 4F); however, FISH analysis did not identify $A L K$ fusion genes (data not shown). At 6 years after his first visit to the hospital (the last follow-up was in November 2016), the patient remains symptom-free, without evidence of recurrence of either tumor (Fig. 3B and C).

This case report was approved by the Ethics Committee of Toyooka Hospital and the patient consented to the publication of the case details and associated images.

\section{Discussion}

High-sensitivity IHC, RT-PCR and FISH have been used to detect fusion genes of ALK in lung cancer (11). The results of high-sensitivity IHC for ALK expression are almost entirely consistent with the results of $A L K$ fusion gene detection by RT-PCR or FISH. However, a small number of NECs express ALK protein on high-sensitivity IHC without $A L K$ fusion genes, including $2.9 \%$ of small-cell carcinomas and $0.9 \%$ of LCNECs (10). We herein presented a case of NEC expressing ALK on high-sensitivity IHC without the presence of $A L K$ fusion genes on FISH.

In the present case, the first NEC exhibited small-cell carcinoma-like characteristics and weakly expressed the ALK protein, while the second NEC exhibited LCNEC-like characteristics and moderately expressed the ALK protein. The expression of the ALK protein by both tumors suggests that the second NEC may have been a metastatic lesion of the first NEC, since the rate of ALK expression is very low among NECs of the lungs. It is conceivable that chemotherapy modified the characteristics of the tumor cells. However, the second NEC arose at a site opposite to the first NEC. Therefore, if the second NEC was indeed a metastatic lesion of the first, it would be unlikely for the patient to have remained cancer-free for a long time following resection of the second NEC without any adjuvant therapies. However, if the second NEC was a de novo cancer, the patient would be more likely to remain cancer-free for a long period, but it would seem unlikely that both cancers were NECs, and that they both expressed ALK. Therefore, it has not been elucidated whether the second NEC was a de novo cancer or a metastatic lesion of the first NEC.

Due to financial reasons and its overall versatility, high-sensitivity IHC is usually performed first to detect genomic alterations of $A L K$. It has been reported that the results of IHC, FISH and RT-PCR are well-correlated regarding the detection of $A L K$ fusion genes. However, a very small number of cancers are ALK-positive on high-sensitivity IHC, but do not harbor $A L K$ fusion genes on FISH. Similar to the study of Nakamura et al, Karlsson et al also investigated fusion genes in LCNECs and large-cell carcinomas (LCs) of the lung; they found no $A L K$ fusion genes in the LCNECs or LCs of the lung by FISH, even in cases that were ALK-positive on high-sensitivity IHC (12). The present case also exhibited a similar pattern of ALK expression.

Recently, Ma et al reported that crizotinib, an ALK inhibitor, is also effective for several adenocarcinomas that were positive for ALK on IHC, but did not harbor $A L K$ fusion genes on FISH (13); they noted that current FISH methods for the detection of $A L K$ fusion genes may not be able to detect all fusion genes of $A L K$. Ma et al also demonstrated that RT-PCR is effective in such cases. However, further examinations are required to confirm this phenomenon. Since high-quality RNA could not be obtained from the formalin-fixed samples in our case, accurate RT-PCR for the detection of $A L K$ fusion genes could not be performed.

We herein presented a case of NEC that was ALK-positive on high-sensitivity IHC, but was negative for fusion gene(s) on FISH. Further investigation is required to elucidate the mechanisms underlying ALK expression in NECs. Additional studies should be focused on elucidating whether ALK inhibitors are effective for NECs that are ALK-positive on high-sensitivity IHC but do not harbor ALK fusion genes on FISH.

\section{Acknowledgements}

The authors would like to thank Ms. H. Ogaki, Mr. K Nagaoka, Mr. T. Kuge, Mr. H. Takenaka and Ms. S. Eriguch of Toyooka Hospital for their expert technical assistance.

\section{References}

1. Morris SW, Kirstein MN, Valentine MB, Dittmer KG, Shapiro DN, Saltman DL and Look AT: Fusion of a kinase gene, ALK, to a nucleolar protein gene, NPM, in non-Hodgkin's lymphoma. Science 263: 1281-1284, 1994

2. Soda M, Choi YL, Enomoto M, Takada S, Yamashita Y, Ishikawa S, Fujiwara S, Watanabe H, Kurashina K, Hatanaka H, et al: Identification of the transforming EML4-ALK fusion gene in non-small-cell lung cancer. Nature 448: 561-566, 2007.

3. Chen Y, Takita J, Choi YL, Kato M, Ohira M, Sanada M, Wang L, Soda M, Kikuchi A, Igarashi T, et al: Oncogenic mutations of ALK kinase in neuroblastoma. Nature 455: 971-974, 2008.

4. Li XQ, Hisaoka M, Shi DR, Zhu XZ and Hashimoto H: Expression of anaplastic lymphoma kinase in soft tissue tumors: An immunohistochemical and molecular study of 249 cases. Hum Pathol 35: 711-721, 2004.

5. Murugan AK and Xing M: Anaplastic thyroid cancers harbor novel oncogenic mutations of the ALK gene. Cancer Res 71: 4403-4411, 2011

6. Takeuchi K, Choi YL, Soda M, Inamura K, Togashi Y, Hatano S, Enomoto M, Takada S, Yamashita Y, Satoh Y, et al: Multiplex reverse transcription-PCR screening for EML4-ALK fusion transcripts. Clin Cancer Res 14: 6618-6624, 2008.

7. Chiari R, Metro G, Iacono D, Bellezza G, Rebonato A, Dubini A, Sperduti I, Bennati C, Paglialunga L, Burgio MA, et al: Clinical impact of sequential treatment with ALK-TKIs in patients with advanced ALK-positive non-small cell lung cancer: Results of a multicenter analysis. Lung Cancer 90: 255-260, 2015.

8. Conklin CM, Craddock KJ, Have C, Laskin J, Couture C and Ionescu DN: Immunohistochemistry is a reliable screening tool for identification of ALK rearrangement in non-small-cell lung carcinoma and is antibody dependent. J Thorac Oncol 8: 45-51, 2013. 
9. Zhou J, Zhao J, Sun K, Wang B, Wang L, Chen X, Zheng J, You Q, Wang X, Ding W and Zhou J: Accurate and economical detection of ALK positive lung adenocarcinoma with semiquantitative immunohistochemical screening. PLoS One 9: e92828, 2014.

10. Nakamura H, Tsuta K, Yoshida A, Shibata T, Wakai S, Asamura H, Furuta $\mathrm{K}$ and Tsuda $\mathrm{H}$ : Aberrant anaplastic lymphoma kinase expression in high-grade pulmonary neuroendocrine carcinoma. J Clin Pathol 66: 705-707, 2013.

11. Takeuchi K, Soda M, Togashi Y, Suzuki R, Sakata S, Hatano S, Asaka R, Hamanaka W, Ninomiya H, Uehara H, et al: RET, ROS1 and ALK fusions in lung cancer. Nat Med 18: 378-381, 2012.
12. Karlsson A, Brunnström H, Lindquist KE, Jirström K, Jönsson $\mathrm{M}$, Rosengren $\mathrm{F}$, Reuterswärd $\mathrm{C}$, Cirenajwis $\mathrm{H}$, Borg $\AA$, Jönsson P, et al: Mutational and gene fusion analyses of primary large cell and large cell neuroendocrine lung cancer. Oncotarget 6: 22028-22037, 2015.

13. Ma D, Wang Z, Yang L, Mu X, Wang Y, Zhao X, Li J and Lin D: Responses to crizotinib in patients with ALK-positive lung adenocarcinoma who tested immunohistochemistry (IHC)-positive and fluorescence in situ hybridization (FISH)-negative. Oncotarget 7: 64410-64420, 2016. 